\title{
FEASIBILITY AND OUTCOMES OF AUTOGENIC RELAXATION TRAINING IN ADDITION TO USUAL PHYSIOTHERAPY FOR STROKE SURVIVORS - A PILOT STUDY
}

\author{
Deepak Thazhakkattu Vasu ${ }^{1}$,Nor Azlin Mohd Nordin ${ }^{1}$,See Xiao $\mathrm{Xu}^{1}$,Shazli Ezzat Ghazali ${ }^{1}$ and Siti \\ Norfadilah Abu Zarim² \\ ${ }^{1}$ Center for Rehabilitation and Special Needs Studies, Faculty of Health Sciences, Universiti Kebangsaan Malaysia, \\ Jalan Raja Muda Abdul Aziz, 50300, Kuala Lumpur. \\ 2Physiotherapy Unit, Department of Medical Rehabilitation Services, Hospital Canselor Tuanku Muhriz, Jalan Yaacob \\ Latif, Bandar Tun Razak, 56000, Cheras, Kuala Lumpur.
}

Corresponding author: Nor Azlin Mohd Nordin

Email: norazlin8@ukm.edu.my

\begin{abstract}
A significant percentage of stroke survivors are reported to have anxiety and depression. Autogenic Relaxation Training $(A R T)$, a psychophysiological self-control therapy which aims to induce relaxation proved to be effective in reducing the anxiety and depression in some health conditions. However, there is lack of studies which evaluated the effects of $A R T$ in the rehabilitation of stroke survivors. The aim of this experimental pilot study is to evaluate the feasibility and outcomes of ART in addition to usual physiotherapy for stroke survivors. A total of 14 sub-acute stroke survivors from a teaching hospital were enrolled in this study. All participants received 20 minutes ART followed by 40 minutes usual physiotherapy once a week and they were requested to carry out the intervention at home for twice per week, for six weeks. Intervention outcomes were assessed using Hospital Anxiety and Depression Scale (HADS-A and HADS-D), Barthel Index (BI), Timed Up and Go (TUG) and EuroQol 5-Dimension 5 Levels (EQ5D5L). Changes in all outcome measures were analysed using paired t-test and Wilcoxon signed rank test, with level of significance set at $p<0.05$. Post-intervention, there is statistically significant reduction of the HADS-A ( $p=0.04), \operatorname{HADS}-D(p=0.02)$, TUG $(p=0.004)$ and EQ5D5L ( $p=0.03)$ scores of the participants. Although not statistically significant, the mean score $\pm S D$ of $B$ increased from $95 \pm 12.5$ to $100 \pm 6.25$. The intervention is feasible and acceptable by the stroke survivors with no adverse events reported. In conclusion, ART in addition to usual physiotherapy is feasible and beneficial in reducing anxiety and depression, and improving functional ability, mobility and quality of life among stroke survivors.
\end{abstract}

Keywords: autogenic relaxation training; physiotherapy; stroke; functional ability, quality of life.

\section{INTRODUCTION}

Stroke is an acute focal injury of the central nervous system related to a cause of vascular origin 1 . According to World Health Organization, stroke is the fourth leading cause of loss of productivity and disability-adjusted life years (DALYs) worldwide, behind only HIV/AIDS, unipolar depression and heart disease ${ }^{2}$. Based on the DALYs documented, stroke is among the top five greatest burden of disease $^{3}$ and top ten causes of hospitalization. The prevalence and incidence of stroke in Malaysia increased drastically over the five years ${ }^{4,5}$.

Medical complications following stroke, which consist of neurological deficit, physical impairments and the psychological complications have high impact on physical functions and recovery ${ }^{6}$. The reduction in the physical function and increased dependency in activities of daily living following stroke adversely affect the psychosocial well-being and quality of life of the stroke survivors ${ }^{6,7}$.

Across the stroke cohorts documented, 29\% of stroke survivors have anxiety and $24 \%$ have depression as identified using hospital anxiety and depression scale ${ }^{8}$. Surprisingly, in Malaysia, a high percentage of the stroke survivors reported to have anxiety (86\%) and depressive symptoms $(88 \%)^{9}$. Psychological consequences of stroke are complex and closely related to the quality of life of stroke survivors due to its long-lasting effect on mood, emotions, social interaction ability with other people, ability to return to work and participation in the daily activities ${ }^{10}$.

Autogenic relaxation training (ART) is thought to be beneficial for stroke survivors who have anxiety and depression ${ }^{11}$. ART is a type of 'psychophysiological self-control therapy' which was developed by a German neurologist, Johannes Herinrich Schultz in the year 1950's. ART uses 'passive concentration' principle refers to the individual concentrating on inner sensations such as the heaviness and warmth of limbs, heartbeat, breathing, abdominal warmth and the cooling of forehead upon receiving certain combination of psycho-physiologically adapted stimuli. This principle does not require a conscious active effort to relax the muscles. ART is recommended as an effective technique to promote relaxation and relieve anxiety and depression, particularly 
for the people with potential neurological and muscular dysfunction ${ }^{12}$.

Past researches have reported that ART have positive effect in reducing anxiety and depression in some health conditions such as multiple sclerosis, breast cancer, and post coronary angioplasty ${ }^{11,13-17}$. However, there is limited evidence and low use of ART in minimizing anxiety and depression following stroke during rehabilitation to date. As a key member of rehabilitation providers, physiotherapists not only play their role in maximizing the stroke survivors' potential of independence in daily living activities via stroke rehabilitation program but should also assist in promoting psychological well-being of the stroke survivors. Thus, suitable physiotherapy interventions combined with ART may be useful to maximize the rehabilitation outcomes.

No studies have examined the effects of ART in addition to usual physiotherapy in managing anxiety and depression of the stroke survivors in stroke rehabilitation until today. Therefore, this study aimed to evaluate the feasibility, acceptability and outcomes of ART in addition to usual physiotherapy on the level of anxiety and depression, mobility, functional ability and quality of life among the stroke survivors. We hypothesized that stroke patients who receive ART in addition to usual physiotherapy would report reduction in their anxiety and depression level, and improvement in functional ability, mobility and quality of life. We also predicted that the combined intervention would be feasible and may be introduced as a therapy option for stroke survivors with signs of anxiety and depression.

\section{METHODOLOGY Study design}

This was a one group experimental pilot study. Ethical approval was granted by the Research Ethics Committee, Universiti Kebangsaan Malaysia (Reference Number: UKM PPI/111.8/JEP-2017074).

\section{Participants}

A group of stroke survivors who were starting physiotherapy at Hospital Canselor Tuanku Muhriz (HCTM), Kuala Lumpur were approached and screened based on a set of inclusion/exclusion criteria. Patients with clinical diagnosis of first ever stroke of two weeks to six months post onset were selected. Exclusion criteria included inability to provide informed consent, having severe cognitive impairment indicated by MoCA (cut-off value of $\geq 26$ ), having co-morbid psychiatric disorder other than an affective disorder (such as psychotic disorder or dementia) and are wheelchair-bound. Out of 20 participants screened, 17 participants fulfilled the criteria and were recruited based on the recommended number of subjects required for a pilot study ${ }^{18}$

\section{Intervention}

Recruited participants were given a weekly supervised ART-added physiotherapy session for a total of six weeks. Each session consisted of 20 minutes of ART followed by 40 minutes of usual physiotherapy. In addition, all participants were required to carry out the ART and a set of home exercise program prescribed by the physiotherapist for two times per week during the six-week intervention.

The ART sessions were conducted and supervised by a physiotherapy researcher who was trained by an ART-certified psychologist with vast experience in using the therapy. Verbal and written explanation about ART was provided to the stroke participants in the first intervention session. During ART session, the participants were positioned in a comfortable lying position in a bed and allowed to close their eyes but not fall asleep while listening to the ART instructions. The participants were requested to focus on various parts of their body and experience them in specific ways such as heaviness of limb, warmth of limb, heartbeat, breathing, warmth of abdomen, warmth and cooling of forehead. The ART sessions were delivered in Bahasa Malaysia being the country's national language, as also requested by majority of the participants. Upon completing the ART, the participants continued usual physiotherapy treatment which was carried out by a designated physiotherapist. In general, the physiotherapy consisted of limb strengthening exercises and functional retraining including gait and balance training.

The overall intervention was carried out in a form of one-to-one session for each participant to enable the therapist and researcher to pay close attention to the participant and assess whether the participant had difficulty in following and practising the intervention. This is important as previous study by Kneebone ${ }^{11}$ reported that it would be difficult to carry out ART in group session because of the variability in the disability level among the participants. For the twiceweekly unsupervised ART sessions at home, each participant was given recorded ART instructions. For this purpose, the original English version of the ART instructions was translated into Bahasa Malaysia by a bilingual researcher.

\section{Assessment of outcome}

Outcome of the intervention was assessed in term of change in anxiety and depression level, mobility, functional ability and quality of life. Assessments were carried out twice; one prior to the intervention (baseline) and another at the end of week six of intervention by an independent researcher. 
Anxiety and depression levels among the participants were assessed using the Hospital Anxiety and Depression Scale (HADS). HADS consists of 14 items, which are divided into two subscales namely anxiety subscale and depression subscale, with each subscale consists of seven items respectively. This test has adequate reliability if retested at a six weeks and more interval $(r=0.70)^{19}$.

The mobility level of the participants was assessed using Timed Up and Go (TUG) test. TUG assess the ability to perform sequential motor tasks relative to rising from a standard chair, walking towards a cone which is three meters away from the chair, turning, walking back and sitting on the chair. TUG shows high inter and intra-reliability $(I C C=0.96, p<0.05)^{20}$. A cut-off point of more than 14 seconds in completing the TUG test indicates the risk of falls among the stroke population ${ }^{21}$.

Barthel Index (BI) was used to assess the ability of the participants to carry out functional tasks. BI consists of 10 common daily living activities which are feeding, bathing, grooming, dressing, bowel control, bladder control, toileting, chair transfer, ambulation and stair climbing. The total score of the index ranges from 0 to 100; higher score indicates higher functional ability level. The $\mathrm{BI}$ has excellent inter-rater reliability for standard administration after stroke ${ }^{12}$.

The participants' quality of life was evaluated using EuroQol-five dimensions-five levels (EQ-5D$5 \mathrm{~L})$, a generic index instrument used to describe and value health states ${ }^{22}$. It is a self-administered questionnaire in two parts. The first part is the EQ-5D descriptive system which consists of five dimensions: mobility, self-care, usual activities, pain/discomfort and anxiety/depression. Score from EQ-5D-5L can be converted into health utility index (HUI) which range from 0 (worst health state) to 1 (best health state). The second part is the EQ visual analogue scale (EQ-VAS) on which the respondents rate their current health status from 0 (worst imaginable) to 100 (best possible). Evidence showed that EQ-5D-5L is a valid generic health outcome measure in patients with acute stroke ${ }^{22}$.

\section{Assessment of feasibility}

The feasibility of this intervention was assessed in terms of the practicality of the intervention delivery via feedbacks from the treating physiotherapist, and the occurrence of adverse effects among the participants. We also determined the acceptability of the intervention by assessing the adherence of the participants to the intervention sessions and through participants' feedback.

\section{Data analysis}

All data collected were analysed using SPSS (Statistical Products and Service Solution) version 23. The demographic data was analysed descriptively and reported as frequency, mean and standard deviation. The feasibility of the intervention was assessed via feedbacks from the participants, physiotherapists, and researchers who were involved in this study. Post-intervention changes in all measures were obtained by comparing the mean (using paired t-test) or median (using Wilcoxon signed rank test) score of the variables, with the $p$ value set at $<0.05$.

\section{RESULTS}

\section{Participants' characteristics}

A total of 20 participants were screened; of these, only 17 participants who were eligible enrolled into the study. During the intervention period, three out of $17(17.64 \%)$ participants were lost to follow up due to been uncontactable $(n=1)$ and rehospitalized for other health issues $(n=2)$. These three participants were not included in the statistical analysis, leaving 14 participants (8 males; 6 females) in total whom were analysed for the intervention outcome.

Table 1 shows the participants' characteristic. The mean age of the participants is $59 \pm 12.12$ years, with the mean duration post stroke of 4.6 \pm 3.78 months. There were three types of stroke diagnosed among the participants, namely haemorrhagic $(n=2)$, ischemic $(n=10)$ and unspecific type of stroke $(n=2)$. There were equal number of participants $(n=7)$ for both left and right sided hemiparesis after stroke.

\section{Outcomes of intervention}

The results in Table 2 show that there are statistical significant differences in the pre and post intervention score of HADS-A $(t=2.235$; $p=0.044)$, HADS-D $(t=2.563 ; p=0.024)$, TUG $(Z=2.900 ; \quad p=0.004)$ and EQ-5D-5L $\quad(Z=2.133$; $p=0.033)$. Although there is no statistical difference between the pre and post intervention score of $\mathrm{BI}(Z=1.725 ; p=0.084)$, the mean score \pm $\mathrm{SD}$ of $\mathrm{BI}$ improved from $95 \pm 12.5$ to $100 \pm 6.25$.

\section{Feasibility and acceptability of the intervention}

The physiotherapist involved commented that the intervention was do-able and one-to-one supervised intervention enables them to pay full attention to each participant. No adverse events occurred during the implementation of supervised ART and usual physiotherapy sessions, as well as unsupervised home sessions.

Adherence to the intervention sessions is overall satisfactory. Of the 17 participants who were recruited for the study, 14 (82\%) successfully 
completed the intervention and only two participants $(12 \%)$ withdrew from the intervention due to unavoidable reasons while one participant was uncontactable mid-way through the intervention. Regarding the ART itself, only two participants reported that they had difficulty in performing passive concentration (feeling the inner sensations) during the initial sessions of the ART. However, these participants were able to focus and follow the ART successfully after several practices.

Compliance to the unsupervised home sessions is also satisfactory. Of 14 participants who completed the trial, only one participant $(7 \%)$ did not carry out unsupervised ART and home exercise program as instructed. Five participants (35\%) carried out the unsupervised intervention less than two times per week over the course of six weeks while more than half $(57 \%)$ of the participants practised unsupervised intervention at home for more than two times per week over the six-week period. Nine participants (64\%) reported that they will continue to use ART in addition to the usual physiotherapy and two participants $(14.3 \%)$ reported that they will use ART with or without carrying home exercise program when necessary. Only three participants (21.4\%) reported that they will not continue ART in addition to usual physiotherapy at home as they felt it was no longer necessary.

Feedbacks from most the participants on the intervention were generally encouraging, for examples "I felt that my mood had improved, less anxious and more confident to walk, go up and down stairs compared to the time I just got stroke"; "my mind is free after listening to ART"; "I feel more comfortable and more willing to move"; "less worry and anxious". Three participants perceived no changes in their mood but noticed improvement in their physical function, for example able to walk more steadily.

Table 1: Participants' characteristics $(n=14)$

\begin{tabular}{lcc}
\hline Variables & Categories & Frequency (\%) \\
\hline Gender & Male & $8(57)$ \\
Stroke type & Female & $6(43)$ \\
& Haemorrhagic & $2(14)$ \\
Affected side & Ischemic & $10(72)$ \\
& Unspecific & $2(14)$ \\
Age (years) & Left & $7(50)$ \\
Time since stroke (months) & Right & $7(50)$ \\
& & Mean (SD) \\
\end{tabular}

Table 2: HADS, BI, TUG, EQ-5D-5L and EQVAS scores at pre and post intervention.

\begin{tabular}{lllll}
\hline & Pre & Post & Difference & $\mathrm{p}$ \\
\hline HADS-A, mean (SD) & $5.71(3.38)$ & $3.86(2.50)$ & $1.85(3.11)$ & $0.044^{*}$ \\
HADS-D, mean (SD) & $7.43(3.18)$ & $5.00(3.18)$ & $2.43(3.54)$ & $0.024^{*}$ \\
BI, median(IQR) & $95.00(12.5)$ & $100(6.25)$ & $5.00(6.25)$ & $0.084^{* *}$ \\
TUG, median(IQR) & $15.20(20.8)$ & $12.40(13.81)$ & $2.80(6.98)$ & $0.004^{* *}$ \\
EQ-5D-5L, median(IQR) & $0.54(0.20)$ & $0.60(0.17)$ & $0.06(0.03)$ & $0.033^{* *}$ \\
EQVAS, mean(SD) & $73.50(13.69)$ & $77.50(14.64)$ & $4.00(9.93)$ & $0.156^{*}$
\end{tabular}

*Paired sample t-test; ${ }^{* *}$ Wilcoxon Signed Rank Test; level of statistical significance $p<0.05$

\section{DISCUSSIONS}

Our study findings provide preliminary evidence that ART in addition to usual physiotherapy may reduce anxiety and depression level among the stroke survivors. We noticed a reduction of $32.4 \%$ of the level of anxiety and $32.71 \%$ of the level of depression among the stroke survivors after six weeks of intervention. This finding is in agreement with a previous study by Kneebone et al. ${ }^{11}$ in investigating the feasibility of setting up a relaxation group protocol to treat post-stroke anxiety in an in-patient acute setting. In their study, there was a significant reduction in the self-reported tension rating (TRCs) scale from the 
pre to post training irrespective of the number of sessions attended by stroke survivors ${ }^{11}$.

The benefit of ART has also been documented in studies involving non-stroke populations. A randomized controlled study which was carried out by Golding et al. ${ }^{13}$ demonstrated the beneficial effect of ART in reducing anxiety level of a group of stroke survivors who live in the community after at least one month of practising ART at a frequency of five times per week. ART has also been proven as a powerful self-help therapy in reducing anxiety and depression among women with breast cancer ${ }^{14}$. In addition, systematic reviews have concluded that ART is effective in reducing the anxiety among individuals who have health problem and improving depression symptoms in both clinically and non-clinically depressed populations ${ }^{15,16}$. These positive effect are brought about by the passive concentration activity during the intervention which enables self-regulation in reducing the excessive autonomic arousal and aids in the relaxation.

After completing the six-week intervention, the mobility level of the stroke survivors in our study improved by $18 \%$. This positive outcome could be mostly due to the effects of task-oriented training that we used in the usual physiotherapy. Effectiveness of task-oriented training in enhancing post-stroke mobility has been established in many past studies and systematic review, for example studies by Rensink and coworkers $^{23}$, Blennerhassett and Dite ${ }^{24}$ and Salbach et al. ${ }^{25}$. We hypothesized that improvement in mood among stroke survivors in our study which resulted from ART has further enhanced the benefit of the task-oriented training.

Our study also showed that quality of life of the stroke survivors improved by nearly $11 \%$ post six weeks of the intervention. Although in different disabled population, this finding supports the previous pilot study by Sutherland ${ }^{17}$ who demonstrated the positive effect of ART on health-related quality of life and the level of depression among people with multiple sclerosis. ART is a self-control and self-regulated technique which provides a way for mastery of experiences and enables the independent practice of the readily learned skills. This makes ART obviously different from other relaxation techniques by eliminating the needs of feedback from psychotherapist or a biofeedback machine and strengthens the independence of the patient and indirectly improves their quality of life $\mathrm{e}^{16}$.

We observed improvement in the functional ability of the stroke survivors after the six-week intervention but this is not statistically significant. The participants recruited are considered to have rather high level of functional ability. The non-significant improvement could be due to a ceiling effect as the $\mathrm{BI}$ is no longer able to detect further change in the functional performance among the least impaired stroke survivors ${ }^{11}$. It would be of interest to explore the further improvements in the functional ability level of high functioning participants by using other suitable measurement tools.

Our study has several limitations. Apart from been a small study which has issue of generalizability of results, lack of a control group caused uncertainty of the effectiveness of the intervention, whether produced by the ART alone or the usual physiotherapy alone or the combined intervention. There was also variability in the frequency of unsupervised sessions among by the participants to enable accurate evaluation of the intervention. An experimental study comparing different intervention arrangements is recommended in further investigation.

\section{CONCLUSION}

ART in addition to usual physiotherapy is feasible and beneficial in reducing anxiety and depression level and improving mobility and quality of life among stroke survivors. The combined intervention is also acceptable by stroke survivors, hence can be applied as a therapy option for stroke patients undergoing rehabilitation. Further studies with a control group are recommended to confirm this study findings.

\section{ACKNOWLEDGEMENT}

We thank all the participants and physiotherapy staffs of HCTM for their support and cooperation throughout the study.

\section{REFERENCES}

1. Hendricks HT, Limbeek JV, Geurts AC, Zwarts MJ. Motor recovery after stroke: A systemic review of the literature. Arch Phys Med Rehabil 2002; 83:1629-1637

2. Sacco RL, Kasner SE, Broderick JP, Caplan LR, Culebras A, Elkind MS, George MG, Hamdan A D, Higashida RT, Hoh BL. An updated definition of stroke for the 21st century a statement for healthcare professionals from the American Heart Association/American Stroke Association. Stroke 2013; 44(7): 2064-2089.

3. World Health Organization. World malaria report. World Health Organization 2008 Ed.

4. Loo KW \& Gan SH. Burden of stroke in Malaysia. International Journal of Stroke 2012;7(2): 165-167. 
5. Aziz ZA, YY Lee, Ngah BA, Sidek NN, I Looi, Hanip MR \& Basri HB. Acute stroke registry Malaysia, 2010-2014: Results from the national neurology registry. Journal of Stroke and Cerebrovascular Diseases 2015;24(12): 2701-2709.

6. Janus-Laszuk B, Mirowska-Guzel D, Sarzynska-Dlugosz I \& Czlonkowska A. Effect of medical complications on the after-stroke rehabilitation outcome. Neurorehabilitation 2017; 40(2): 223-232.

7. Kawecka-Jaszcz K, Klocek M, TobiaszAdamczyk B \& Bulpitt CJ. Health-related quality of life in cardiovascular patients. Springer; 2013.

8. Broomfield NM, Quinn TJ, Abdul-Rahim AH, Walters MR \& Evans JJ. Depression and anxiety symptoms post-stroke/TIA: prevalence and associations in crosssectional data from a regional stroke registry. BMC Neurology 2014; 14(1): 1.

9. Mohd Zulkifly MF, Ghazali SE, Normah CD \& Subramaniam P. The Influence of demographic, clinical, psychological and functional determinants on post-stroke cognitive impairment at day care stroke center, Malaysia. Malaysian Journal of Medical Sciences 2016 ;23(2): 53-64.

10. Knapp P, Young J, House A \& Forster A. Non-drug strategies to resolve psychosocial difficulties after stroke. Age and ageing 2000; 29(1): 23-30.

11. Kneebone I, Walker-Samuel N, Swanston J \& Otto E. Relaxation training after stroke: potential to reduce anxiety. Disability and rehabilitation 2014;36(9): 771-774.

12. Sarker SJ, Rudd AG, Douiri A \& Wolfe CD. Comparison of 2 extended activities of daily living scales with the Barthel index and predictors of their outcomes. Stroke 2012; 43(5): 1362-1369.

13. Golding K, Kneebone I \& Fife-Schaw C. Self-Help relaxation for post-stroke anxiety: A randomised, controlled pilot study. Clinical rehabilitation 2016 ;30(2):174-80.

14. Hidderley $M \&$ Holt MA. Pilot randomized trial assessing the effects of autogenic training in early stage cancer patients in relation to psychological status and immune system responses. European Journal of Oncology Nursing 2004; 8(1): 61-65.
15. Manzoni GM, Pagnini F, Castelnuovo G \& Molinari E. Relaxation training for anxiety: A ten-years systematic review with meta-analysis. BMC psychiatry 2008; 8(1): 1.

16. Morgan AJ \& Jorm AF. Self-Help interventions for depressive disorders and depressive symptoms: A systematic review. Annals of general psychiatry 2008; 7(1): 1.

17. Sutherland G, Andersen MB \& Morris T. Relaxation and health-related quality of life in multiple sclerosis: the example of autogenic training. Journal of behavioral medicine 2005; 28(3): 249-256.

18. Billingham SA., Whitehead AL \& Julious SA. An audit of sample sizes for pilot and feasibility trials being undertaken in the United Kingdom registered in the United Kingdom Clinical Research Network database. BMC Medical Research Methodology 2013;13(1):1.

19. Stern AF. Questionnaire review: The hospital anxiety and depression scale. Occupational Medicine 2014;64:393-394.

20. Faria CD, Teixeira-Salmela LF, Neto MG \& Rodrigues-de-Paula F. Performance-based tests in subjects with stroke: outcome scores, reliability and measurement errors. Clinical rehabilitation 2012; 26(5): 460-469.

21. Andersson $\AA$, Kamwendo $K$, Seiger A \& Appelros P. How to identify potential fallers in a stroke unit: validity indexes of 4 test methods. Journal of rehabilitation medicine 2006;38(3): 186-191.

22. Golicki D, Niewada M, Buczek J, Karlińska A, Kobayashi A, Janssen M \& Pickard AS. Validity of EQ-5D-5L in stroke. Quality of life research 2015 ;24(4): 845-850.

23. Rensink M, Schuurmans M, Lindeman E \& Hafsteinsdottir T. Task-oriented training in rehabilitation after stroke: systematic review. Journal of advanced nursing 2009;65(4): 737-754.

24. Blennerhassett J \& Dite W. Additional task-related practice improves mobility and upper limb function early after stroke: A randomised controlled trial. Australian Journal of Physiotherapy 2004; 50(4): 219-224.

25. Salbach NM, Mayo NE, RobichaudEkstrand S, Hanley JA, Richards CL \& 
Wood-Dauphinee S. The effect of a taskoriented walking intervention on improving balance self-efficacy poststroke: A randomized, controlled trial. Journal of the American Geriatrics Society 2005; 53(4): 576-582. 